Review

\title{
Optimization of the Ultrasound-Induced Blood-Brain Barrier Opening
}

\author{
Elisa E. Konofagou ${ }^{1,2}$ \\ 1. Department of Biomedical Engineering, Columbia University, New York, NY; \\ 2. Department of Radiology, Columbia University, New York, NY.
}

\author{
$\bowtie$ Corresponding author: ek2191@columbia.edu.
}

( ) Ivyspring International Publisher. This is an open-access article distributed under the terms of the Creative Commons License (http://creativecommons.org/ licenses/by-nc-nd/3.0/). Reproduction is permitted for personal, noncommercial use, provided that the article is in whole, unmodified, and properly cited.

Received: 2012.II.2I; Accepted: 2012.12.29; Published: 2012.12.3।

\begin{abstract}
Current treatments of neurological and neurodegenerative diseases are limited due to the lack of a truly non-invasive, transient, and regionally selective brain drug delivery method. The brain is particularly difficult to deliver drugs to because of the blood-brain barrier (BBB). The impermeability of the BBB is due to the tight junctions connecting adjacent endothelial cells and highly regulatory transport systems of the endothelial cell membranes. The main function of the BBB is ion and volume regulation to ensure conditions necessary for proper synaptic and axonal signaling. However, the same permeability properties that keep the brain healthy also constitute the cause of the tremendous obstacles posed in its pharmacological treatment. The BBB prevents most neurologically active drugs from entering the brain and, as a result, has been isolated as the rate-limiting factor in brain drug delivery. Until a solution to the trans-BBB delivery problem is found, treatments of neurological diseases will remain impeded. Over the past decade, methods that combine Focused Ultrasound (FUS) and microbubbles have been shown to offer the unique capability of noninvasively, locally and transiently open the BBB so as to treat central nervous system (CNS) diseases. Four of the main challenges that have been taken on by our group and discussed in this paper are: I) assess its safety profile, 2) unveil the mechanism by which the BBB opens and closes, 3 ) control and predict the opened BBB properties and duration of the opening and 4) assess its premise in brain drug delivery. All these challenges will be discussed, findings in both small (mice) and large (non-human primates) animals are shown and finally the clinical potential for this technique is shown.
\end{abstract}

Key words: blood-brain barrier, drug delivery, focused ultrasound, mechanism,microbubble.

\section{Introduction}

\section{The blood-brain barrier (BBB) physiology: Structure and Function}

The BBB is a specialized substructure of the vascular system consisting of endothelial cells connected together by tight junctions, the pericytes as well as astrocytes [1]. The luminal and abluminal membranes line the inner wall of the vessel and act as the permeability barrier (Fig. 1(b)). The combination of tight junctions and these two membranes characterizes the BBB as having low permeability to large and ionic substances. However, certain molecules such as glucose and amino acids are exceptions, because they are actively transported. It has also been shown that lymphocytes can traverse the BBB by going through temporarily opened tight junctions of the endothelial walls. The astrocytes have been proven to offer a protective mechanism of the neurons to any mechanical effect [1-3].

\section{The BBB and neurotherapeutics}

Several neurological disorders remain intracta- 
ble to treatment by therapeutic agents because of the BBB, the brain's natural defense. By acting as a permeability barrier, the BBB impedes entry from blood to the brain of virtually all molecules with higher than $400 \mathrm{Da}$ of molecular weight, thus rendering many potent neurologically active substances and drugs ineffective simply because they cannot be delivered to where they are needed. As a result, traversing the BBB remains the rate-limiting factor in brain drug delivery development ([1]).

\section{Focused Ultrasound (FUS)}

Focused ultrasound (FUS) utilizes the same concept of acoustic wave propagation as the more widely known diagnostic ultrasound applications. However, instead of acquiring and displaying echoes generated at several tissue interfaces for imaging, FUS employs concave transducers that usually have either a single geometric focus or use phased arrays to electronically steer it, at which most of the power is delivered during sonication in order to induce mechanical effects, thermal effects, or both.

\section{BBB opening using FUS and microbubbles}

Blood-brain barrier opening induced by ultrasound at or near ablation intensities was first observed some accompanied by neuronal damage [5-7] and some not [8]. After reducing the acoustic intensity and duty cycle, BBB opening was still observed, but without the macroscopic damage [9]. With the addition of intravenously(IV)-injected microbubbles prior to sonication, BBB opening was determined to be transient [10] in the presence of Optison ${ }^{\mathrm{TM}}$ (Optison $^{\mathrm{TM}}$; Mallinckrodt Inc., St. Louis, MO), which are albumin-coated, octafluoropropane-filled microbubbles of $3-4.5 \mu \mathrm{m}$ in diameter and are usually used to enhance blood vessels on clinical ultrasound images through opacification. The BBB opening procedure could also be monitored with MRI and MR contrast agents [10]. This showed the potential of opening the BBB without damaging parenchymal cells, such as neurons. Further investigation entailed study of this phenomenon with Optison ${ }^{\mathrm{TM}}$ to search for a difference in threshold of BBB opening and neuronal damage, assess the mechanism of the opening in rabbits with [11-13] or without [14] a craniotomy and skull heating in pigs [12]. The advantage of having microbubbles present in the blood supply is that it allows for the reduction of the ultrasound intensity, avoidance of thermal effects, and the reduction of the likelihood of irreversible neuronal damage [11-21]. Although there are many indications that damage can be contained to minimal hemorrhage [16], the complete safety profile remains to be assessed. In addition, indications to various mechanisms such as the dilation of vessels, temporary ischemia, mechanically induced opening of the tight junctions, and the activation of various transport mechanisms have been reported $[9,13,18]$.

There are several reports over the past decade using FUS and microbubbles to disrupt the blood-brain barrier but this paper will focus on the main findings by our group given the brevity required. Our group has demonstrated feasibility of BBB opening through intact skull and skin and successful imaging of the BBB opening in the area of the hippocampus at sub-millimeter imaging resolution using a 9.4T MR scanner in both wildtype $([19,21-23])$ and Alzheimer's mice ([24]). Our group also concentrates on a specific brain region (e.g., the hippocampus), which is key in neurodegenerative disease, such as Alzheimer's, and can be successfully and reproducibly targeted ([25]). Delivery of molecules of up to $2000 \mathrm{kDa}$ in molecular weight was also demonstrated ([26]). Preliminary histology indicated no structural damage in the area of the hippocampus ([27]). Finally, it is important to note that the microbubbles used for BBB opening have been approved by the Food and Drug Administration (FDA) for human use in contrast echocardiography, e.g., for the detection of myocardial infarction ([28]). It is equally important to specify that the pressure amplitudes used for BBB opening are of similar range to ultrasound diagnostic levels $(<1.5-2 \mathrm{MPa})$ and, therefore, assumed safe for human use ([29]) while the pulse duration is by orders of magnitude longer.

\section{Microbubbles in contrast ultrasound and associated bioeffects}

Currently, in the U.S., microbubbles are only FDA-approved for echocardiography in patients with sub-optimal images of the cardiac chambers. However, microbubbles have shown promise for imaging myocardial perfusion using intermittent contrast destruction pulses. Therefore, most in vivo bioeffects studies have focused on the heart ([30]). For a given frequency, separate pressure thresholds exist for microbubble destruction and the onset of bioeffects ([31];[32, 33]). Safe cardiac perfusion imaging would then be done with the microbubble clearance pulse being between these thresholds. Extravascular drug delivery to the brain would then be performed near the threshold for transient opening, but well below the conditions for permanent damage. Human doses for commercially available microbubbles used in contrast echocardiography could provide a useful benchmark for therapy trials. However, the human dose for imaging purposes varies widely. A typical dose ranges between 6-12 x $10^{7}$ microbubbles per $\mathrm{kg}$ 
(60-120 microbubbles per mg). Mean diameters are given, but detailed information of the polydispersed size distributions is lacking. Thus, the effects of microbubble size and concentration on safety are difficult to decouple from previous studies using these commercial agents. Our ability to generate and isolate microbubbles of distinct and narrow size distributions with well defined concentrations has allowed for to probing these effects in the studies described.

Several studies have shown an increase in bioeffects with increasing microbubble dose. For Definity and Optison, increases in rat cardiomyocyte cell death and premature heart beats and microvessel leakage were found after insonation [33, 37]. Similar dose-response relationships have been observed for BBB opening ([34], [35], Yang [36] 7). [37] compared insonation of Optison, Definity and Imagent in the rat heart and found that microvascular effects were similar when expressed as the number of microbubbles injected. They concluded that shell type and encapsulated gas have little effect on bioeffects. Given the polydispersed size distribution of the different formulations, however, the effects of size are difficult to glean from that study. However, little is known about the effects of microbubble size on bioeffects. Christiansen et al. [38] found that intra-arterial injection was more effective than intravenous injection for gene transfection through sonoporation. This result was attributed to the difference in microbubble sizes delivered to the insonified region. Several biophysical studies have shown remarkable size dependence for microbubble oscillation and destruction ([39]; [40]).

\section{Clinical Relevance of BBB disruption}

\section{I. Neurodegenerative disease}

According to the 2008 US News Health report, over 4 million U.S. men and women suffer from Alzheimer's disease; 1 million from Parkinson's disease; 350,000 from multiple sclerosis; and 20,000 from ALS. Worldwide, these four diseases account for more than 20 million patients. Although great progress has been made in recent years toward understanding of neurodegenerative diseases like Alzheimer's, Parkinson's, multiple sclerosis, ALS and others, few effective treatments and no cures are currently available. Aging greatly increases the risk of neurodegenerative disease and the average age of Americans is steadily increasing. Today, over 35 million Americans are over the age of 65 . Within the next 30 years this number is likely to double, putting more and more people at increased risk of neurodegenerative disease. Alzheimer's disease, which has emerged as one of the most common brain disorders, begins in the hippo- campal formation and gradually spreads to the remaining brain at its most advanced stages, and is characterized partly by deposition of amyloid plaques in the brain tissue but also in the blood vessels themselves ([41]). For the purpose of this study, we will focus on the treatment of Alzheimer's disease through the FUS-induced blood-brain barrier opening and therefore, the targeted region in the brain will be the hippocampus.

\subsection{Drug delivery in neurodegenerative disease}

Over the past decade, numerous small- and large-molecule products have been developed for treatment of neurodegenerative diseases with mixed success. When administered systemically in vivo, the BBB inhibits their delivery to the regions affected by those diseases. A review of the Comprehensive Medicinal Chemistry database indicates that only $5 \%$ of the more than 7000 small-molecule drugs treat the Central Nervous System (CNS) [4]. With these, only four CNS disorders can be treated: depression, schizophrenia, epilepsy, and chronic pain [42]; [43] . Despite the availability of pharmacological agents, potentially devastating CNS disorders and age-related neurodegenerative diseases, such as Alzheimer's disease, Parkinson's disease, Huntington's disease, multiple sclerosis, and amythrophic lateral sclerosis (ALS), remain undertreated mainly because of the impermeability of the BBB $([1,4])$. One of the goals of our studies has been to optimize the FUS method and elucidate the physical mechanism in order to ultimately deliver therapeutics to the brain and significantly facilitate treatment of currently intractable and devastating neurodegenerative diseases. As indicated before, there has been several hypotheses and reports on the physiological mechanism $[13,55,58]$, but the physical mechanism has been progressively unveiled and identified as being solely related to stable cavitation at low pressures $([17,60,64$, 69]) and a combination of stable and inertial at higher pressures ([60, 64]) (Fig. 7(iii)).

A successful drug delivery system requires transient, localized, and noninvasive targeting of a specific tissue region. None of the current techniques clinically used, or currently under research, address these issues within the scope of the treatment of neurodegenerative diseases. As a result, the present situation in neurotherapeutics enjoys few successful treatments for most CNS disorders. Some of those routes of administration are listed in Table 1. Several pharmaceutical companies use the technique known as lipidization, which is the addition of lipid groups to the polar ends of molecules to increase the permeability of the agent [44]. However, the effect is not lo- 
calized as the permeability of the drug increases not only in the targeted region, but over the entire brain and body. There can thus be a limit to the amount absorbed before the side-effects become deleterious [44].

A second set of techniques under study are neurosurgically-based drug delivery methods, which involve the invasive implantation of drugs into a region by a needle [45]; [46]. The drug spreads through diffusion and is localized to the targeted region, but diffusion does not allow for molecules to travel far from their point of release. In addition to this, invasive procedures traverse untargeted brain tissue potential causing unnecessary damage. Other techniques utilize solvents mixed with drugs or adjuvants (pharmacological agents) attached to drugs to disrupt the BBB through dilation and contraction of the blood vessels $[1,4,47]$. However, this disruption is not localized within the brain, and the solvents and adjuvants used are potentially toxic. This technique may constitute a delivery method specific to the brain, but it requires special attention to each type of drug molecule and a specific transport system resulting in a time-consuming and costly process while still not being completely localized to the targeted region. FUS in combination with microbubbles constitutes thus the only truly transient, localized, and noninvasive technique for opening the BBB. Due to these unique advantages over other existent techniques (Table 1), FUS may facilitate the delivery of already developed pharmacological agents and could significantly impact how devastating CNS diseases are treated.

However, despite the fact that FUS is currently the only technique that can open the BBB locally and noninvasively, several key aspects of this phenomenon remain unexplored. A clear correlation of BBB opening with microbubbles has been shown [10, 17, 19]. Although the presence of microbubbles allows for a reduction in the necessary acoustic pressure for BBB opening, it also allows for the possibility of disrupting the microbubble through inertial cavitation [47-49]. The resulting effects can not only open the tight junctions, but also could induce irreversible damage to the blood vessels and its surrounding cells [27]. Recent studies have indicated that BBB opening may occur without necessarily incurring inertial cavitation, without [14] or with [17] craniotomy. However, it is not clear how the different types of mechanical effects lead to BBB opening and how the role of the microbubble can be optimized. Given the strong coupling of microbubble size and concentration to the response to insonation, a mechanistic study to BBB opening by contrast-assisted focused ultrasound must include these parameters. Control over both ultrasound and microbubble parameters is essential for the proper optimization and understanding of the FUS technique. However, to our knowledge no study to date has included a thorough investigation of both of these components.

Table I. Techniques shown to induce trans-BBB transport or BBB disruption.

\begin{tabular}{|c|c|c|c|c|}
\hline Lipidization & $\begin{array}{l}\text { Lipidize the drug. } \\
\text { Allows uptake in the BBB. }\end{array}$ & $\begin{array}{l}\text { Increases penetration across all biological mem- } \\
\text { branes. }\end{array}$ & Yes & No \\
\hline $\begin{array}{l}\text { Transcranial brain drug } \\
\text { delivery }\end{array}$ & $\begin{array}{l}\text { Neurosurgically-based drug delivery method. } \\
\text { Diffusion-based method. }\end{array}$ & $\begin{array}{l}\text { Invasive. Diffusion reduces the initial concentration } \\
\text { by } 90 \% \text { when traveling only } 0.5 \mathrm{~mm} \text {. }\end{array}$ & No & Yes \\
\hline $\begin{array}{l}\text { Solvent/adjuvant-mediated } \\
\text { BBB disruption }\end{array}$ & $\begin{array}{l}\text { Solvent and adjuvants disrupt the BBB using } \\
\text { dilation, contraction, and other methods. }\end{array}$ & Disrupts the BBB in all of the brain. Potentially toxic. & Yes & No \\
\hline $\begin{array}{l}\text { Delivery through endoge- } \\
\text { nous transporters }\end{array}$ & $\begin{array}{l}\text { Use endogenous transporters to traverse the } \\
\text { BBB. }\end{array}$ & $\begin{array}{l}\text { Requires medicinal chemistry to modify drugs and } \\
\text { knowledge of the endogenous transporters. }\end{array}$ & Yes & No \\
\hline Ultrasound & Focused Ultrasound (FUS) with microbubbles & Possible irreversible damage may be induced. & Yes & Yes \\
\hline
\end{tabular}

\subsection{FUS-facilitated BBB opening in drug de- livery for treatment of neurodegenerative disease}

Realizing the strong premise of this technique for facilitation of drug delivery to specific brain regions, we showed that the BBB can be opened reliably and reproducibly in the hippocampal region in mice[19-26, 50-52]. By developing a better understanding of the underlying physical parameters that are responsible for the opening of the $\mathrm{BBB}$, namely, the ultrasound and microbubble parameters, we will be in a position to fully exploit this methodology and to do so safely. The feasibility of the technique at optimized ultrasound and microbubble parameters for reversible BBB opening, as determined in vivo, has been tested on wild-type mice as a first step to identify the potential of this technique in the treatment of neurodegenerative diseases ([24]). The MR imaging methods developed allow for high sensitivity, high 
spatial resolution, and high temporal resolution. The latter is achieved through the slow diffusion of intraperitoneally-injected gadolinium. The added potential of combining this ultrasound technique with any therapeutic agent may renew possibilities in potentially employing available pharmacological agents, whose development has currently been abandoned because of poor BBB penetration. This may thus result in the novel and effective treatment of several, potentially devastating, neurological and neurodegenerative diseases. As indicated above, we will concentrate on the feasibility of noninvasive and localized treatment Alzheimer's disease by specifically targeting the hippocampus. However, the FUS technique can, in principle, be combined and applied in the case of any neurological disease. Therefore, findings of this study may not only impact treatment of a specific disease but also the entire field of brain diseases. In summary, FUS stands to make an important impact in the brain drug delivery warranting thus its optimization through understanding of the type of interaction between the microbubble, the tissue and the FUS beam.

\section{Drug delivery through the opened BBB}

The delivery of many large agents using focused ultrasound (FUS) and microbubbles has been demonstrated in previous studies by our group and others: MRI contrast agents such as Omniscan (573 Da) [22] and Magnevist ${ }^{\circledR}$ (938 Da) [21], Evans Blue [53], Trypan Blue [54], Herceptin (148 kDa) [53], horseradish peroxidase (40 kDa) [55], doxorubicin (544 Da) [56], multi-sized Dextran [26], rabbit anti-A $\beta$ antibodies [54] and stem cells [70]. Despite the promise shown by the delivery of such a variety of compounds, several questions with the effectiveness of the delivery remain. In particular, it is still not known whether therapeutic molecules can cross through the BBB opening into the intracellular neuronal space so that they can trigger the required downstream effects for neuronal regeneration.

\section{METHODS AND RESULTS}

\section{Methods for Inducing and Assessing BBB Opening}

\section{I.I FUS and microbubbles}

The experimental setup is shown in Fig. 1(a). The FUS transducer (center frequency: $1.5 \mathrm{MHz}$; focal depth: $60 \mathrm{~mm}$; outer radius: $30 \mathrm{~mm}$; inner radius 11.2 mm, model: cdc7411-3, Imasonic, Besançon, France) is used to perform sonication immediately following bubble administration. The transducer is driven by a function generator (Agilent Technologies, Palo Alto,
CA, USA) through a 50-dB power amplifier (ENI Inc., Rochester, NY, USA). A cone filled with degassed and distilled water is attached to the transducer system. The transducer is attached to a computer-controlled positioner (Velmex Inc., Bloomfield, NY). The PCD, a 5-cm cylindrically focused broadband hydrophone (Sonic Concepts, Bothell, WA, USA), with a cylindrical focal region (height $19 \mathrm{~mm}$, diameter $3.64 \mathrm{~mm}$ ) is placed at 600 from the longitudinal axis of the FUS beam. The PCD and the FUS transducer are confocally aligned. The acoustic emissions from the microbubbles are captured with the PCD and collected using a digitizer (model 14200, Gage Applied Technologies, Inc., Lachine, $\mathrm{QC}$, Canada) through a $20 \mathrm{~dB}$ amplifier (model 5800, Olympus NDT, Waltham, MA, USA). Microbubbles (Definity ${ }^{\circledR}$ : mean diameter range: 1.1-3.3 $\mu \mathrm{m}$, Lantheus Medical Imaging, MA, USA, or lipid-shelled microbubbles manufactured in-house and size-isolated using differential centrifugation [57]) are activated and used within $24 \mathrm{~h}$ after activation. Following activation, a 1:20 dilution solution is prepared using $1 \mathrm{x}$ phosphate-buffered saline (PBS) and slowly injected into the tail vein $(1 \mu \mathrm{l}$ per gram of mouse body weight). Both transducers use pulsed-wave FUS (burst rate: $10 \mathrm{~Hz}$; burst duration: 20 ms; duty cycle: $20 \%$ ) in two 30 -s sonication intervals with a 30 -s intermittent delay. Peak-rarefactional acoustic pressures of $0.15,0.30,0.45$ and $0.60 \mathrm{MPa}$ are typically used as they have been shown to provide the best tradeoff between safety and BBB opening [27]. One side of the hippocampus in the horizontal orientation is sonicated in each mouse. Acoustic parameters other than the pressure have also been studied with respect to their role in BBB disruption. One of those is the pulse length [58]. In that study, mouse brains were pulse sonicated (center frequency: 1.5 $\mathrm{MHz}$, peak-negative pressure: $0.3 \mathrm{MPa}$, pulse length $(\mathrm{PL})$ : 0.002-30 ms, pulse repetition frequency (PRF): $6.25,25,100 \mathrm{kHz}$ ) continuously or with a burst length of 1000 pulses (burst repetition frequency (BRF): 0.1, 1 , 2 , or $5 \mathrm{~Hz}$ ) through the intact scalp and skull for 11 $\mathrm{min}$. One minute after the start of sonication, fluorescence-tagged dextran $(60 \mu \mathrm{g} / \mathrm{g}$, molecular weight: 3 $\mathrm{kDa})$ and Definity ${ }^{\circledR}$ microbubbles $(0.05 \mu \mathrm{l} / \mathrm{g})$ were intravenously injected. After $20 \mathrm{~min}$ of circulation, the mice were transcardially perfused, and the brains were sectioned and imaged using fluorescence microscopy. In order to determine the microbubble size dependence, mice have been injected intravenously with lipid-shelled bubbles of either 1-2, $4-5$ or $6-8 \mu \mathrm{m}$ in diameter while the concentration was $10^{7}$ numbers/mL [50]. 

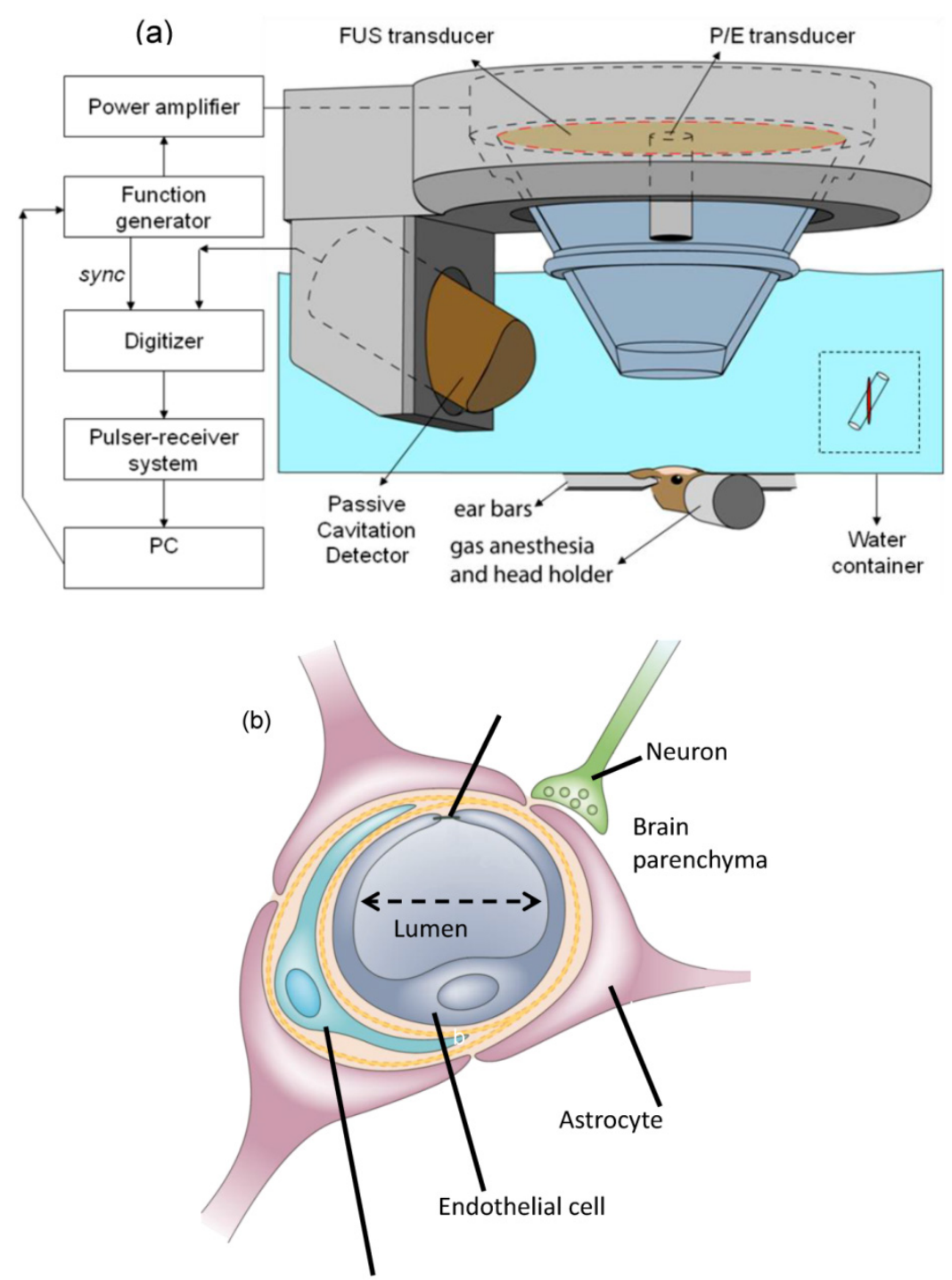

FIGURE I. a) Block diagram and illustration of the experimental setup. The PCD was positioned at 60 。 relative to the longitudinal axis of the FUS beam. The overlap between the focal regions of PCD (blue) and FUS (red) occurring inside the murine brain is illustrated in the inset; b) Lateral cross-section of a brain capillary: the micron-sized bubbles are flowing in the lumen and oscillate when activated by the FUS beam.

\section{I.2. Magnetic resonance imaging}

A vertical-bore 9.4T MR system (Bruker Biospin, Billerica, MA, USA) was used to confirm the blood-brain barrier opening in the murine hippocampus. Each mouse was anesthetized using 1-2\% of isoflurane gas and was positioned inside a single resonator. The respiration rate was monitored throughout the procedure using a monitoring or gating system (SA Instruments Inc., Stony Brook, New York, USA). Prior to introducing the mouse into the scanner, intraperitoneal (IP) catheterization was performed. Two different protocols were used for MR imaging. The first protocol was a three-dimensional (3D), T1-weighted SNAP gradient echo pulse sequence, which acquired horizontal images using $\mathrm{TR} / \mathrm{TE}=20 / 4 \mathrm{~ms}$, a flip angle of $25 \mathrm{deg}$, NEX of 5 , a total acquisition time of $6 \mathrm{~min}$ and $49 \mathrm{~s}$, a matrix size of $256 \times 256 \times 16$ pixels and a field of view (FOV) of $1.92 \times 1.92 \times 0.5 \mathrm{~cm}^{3}$, resulting in a resolution of $75 \times 75 \times 312.5 \mu^{3}$. The second protocol was a $3 \mathrm{D}$ T2*-weighted GEFC gradient echo pulse sequence, which acquired horizontal images using $\mathrm{TR} / \mathrm{TE}=20 / 5.2 \mathrm{~ms}$, a flip angle of $10 \mathrm{deg}$, NEX of 8 , a total acquisition time of $8 \mathrm{~min}$ and $12 \mathrm{~s}$, a matrix size of $256 \times 192 \times 16$ pixels and a FOV of $2.25 \times 1.69 \times 0.7 \mathrm{~cm}^{3}$, resulting in a resolution of $88 \times 88 \times 437.5 \mu \mathrm{m}^{3}$. Both protocols were applied approximately $30 \mathrm{~min}$ after IP injection of $0.30 \mathrm{ml}$ of gadodiamide $(590 \mathrm{Da}$, Omnis$\operatorname{can}^{\circledR}$, GE Healthcare, Princeton, NJ, USA), which allowed sufficient time for the gadodiamide to diffuse into the sonicated region. 


\section{I.3. Acoustic emission signal acquisition and analysis}

The acoustic emission signals acquired by the PCD are sampled at $25 \mathrm{MHz}$ to accommodate the highest memory limit of the digitizer involved in each case. A customized spectrogram function (30-cycles, i.e., $20 \mu$ s, Chebyshev window; 95\% overlap; 4096-point FFT) in MATLAB $^{\circledR}$ (2007b, Mathworks, Natick, MA) is used to generate a time-frequency map, which provided the spectral amplitude in time. The spectrogram can then clearly indicate how the frequency content of a signal changes over time. Therefore, the onset of the broadband response and its duration could be clearly demonstrated on the spectrogram.

The acoustic emissions are quantified in vivo. A high-pass, Chebyshev type 1, filter with a cut-off of 4 $\mathrm{MHz}$ was first applied to the acquired PCD signal. The acoustic emission collected by the focused hydrophone was used in the quantification of the ICD, the harmonic (nf, $\mathrm{n}=1,2, \ldots, 6)$, sub-harmonic (f/2) and ultra-harmonics $(\mathrm{nf} / 2, \mathrm{n}=3,5,7,9)$ frequencies produced by stable cavitation [59] were filtered out by excluding $300-\mathrm{kHz}$ bandwidths around each harmonic and $100-\mathrm{kHz}$ bandwidths around each sub- and ultra-harmonic frequency. These bandwidths were designed to filter for the broadband response and to ensure that the stable cavitation response was not included in the ICD calculation. The root mean square (RMS) of the spectral amplitude ( $\mathrm{V}_{\mathrm{RMS}}$ ) could then be obtained from the spectrogram after filtering. To maximize the broadband response compared to the sonication without microbubbles, only the first $50 \mu \mathrm{s}$ of sonication were considered in the ICD calculation, which was performed by integrating the $\mathrm{V}_{\mathrm{RMS}}$ varia- tion within an interval of $0.75 \mu$ s (i.e., calculating the area below the $V_{\text {RMS }}$ curve between $0.095 \mathrm{~ms}$ and 0.145 $\mathrm{ms})$. In order to remove the effect of the skull in the ICD calculation, the $V_{\text {RMS }}$ in the case without microbubbles was also calculated and was subtracted from the results with the microbubbles to obtain the net bubble response. A Student's $t$-test was used to determine whether the ICD was statistically different between different pressure amplitudes. A P-value of $P<0.05$ was considered to denote a significant difference in all comparisons.

\section{Acoustic parameter dependence and mechanism of BBB opening}

We have found that the peak rarefactional pressure and the microbubble diameter dictate the physical mechanism, i.e., whether the BBB opening occurs in the presence of stable or inertial cavitation [60]. The BBB opening pressure threshold is identified to fall between 0.30 and $0.45 \mathrm{MPa}$ in the case of the 1-2- $\mu \mathrm{m}$ bubbles with inertial cavitation while BBB opening occurs with stable cavitation only at pressures between 0.15 and $0.30 \mathrm{MPa}$ in the $4-5$ and $6-8-\mu \mathrm{m}$ cases $[50,60]$. At every acoustic pressure, both the region of contrast enhancement in the MRI imaging and the amplitude of broadband emissions increased with the bubble diameter. The IC threshold is found to be bubble size independent and to lie between $0.30 \mathrm{MPa}$ and $0.45 \mathrm{MPa}$ for all bubble sizes (Fig. 2). The underlying reason for this independence of the threshold on the bubble size is currently being investigated. In fluorescence imaging, the PL of $2.3 \mu$ s was found to be sufficient for BBB opening and Dextran delivery (Fig. $3)$.

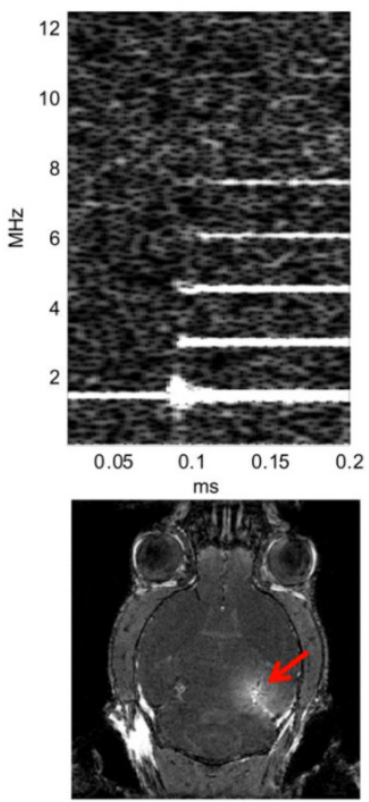

a
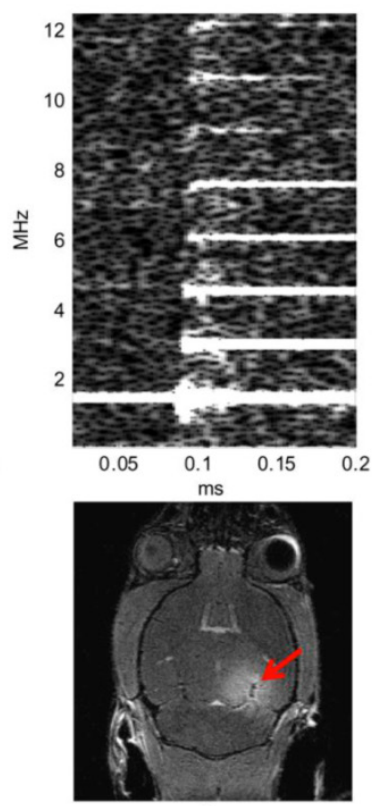

b
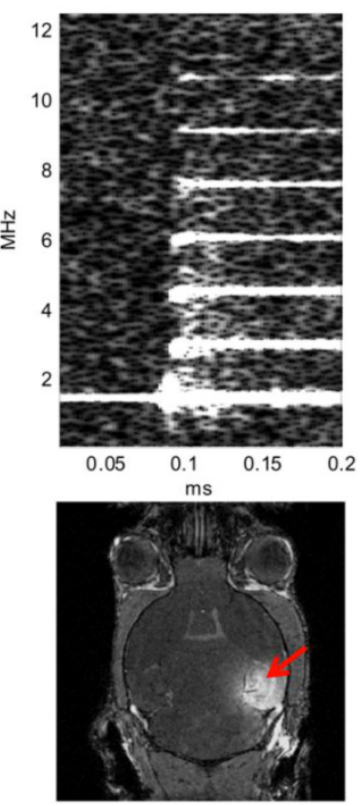

C
FIGURE 2. Spectrogram during the first $0.2 \mathrm{~ms}$ sonication. Broadband acoustic emissions were detected at (b) $0.45 \mathrm{MPa}$ and (c) $0.60 \mathrm{MPa}$ but not at (a) $0.30 \mathrm{MPa}$. Corresponding MRI images confirm that the blood-brain barrier (BBB) could be opened at $0.30 \mathrm{MPa}$, i.e., without inertial cavitation $[60,64]$. The red arrows indicate the location of BBB opening which is the hippocampus. 


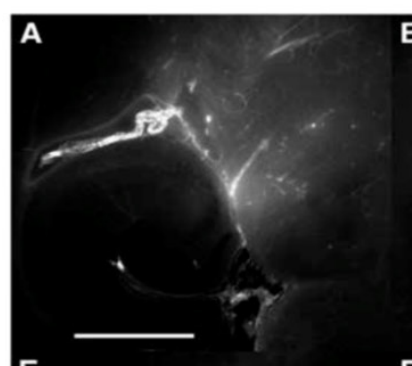

E

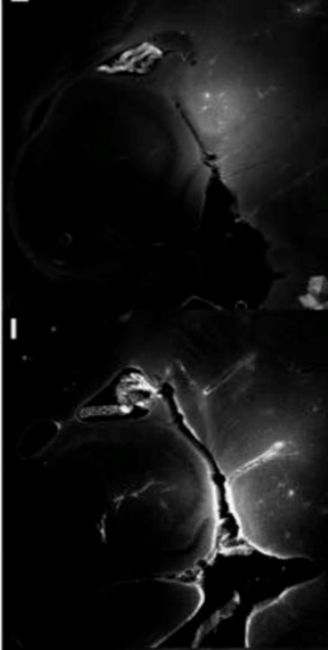

M

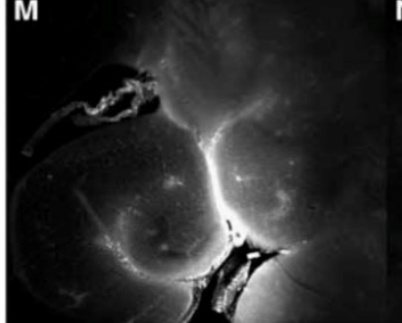

$\mathbf{F}$

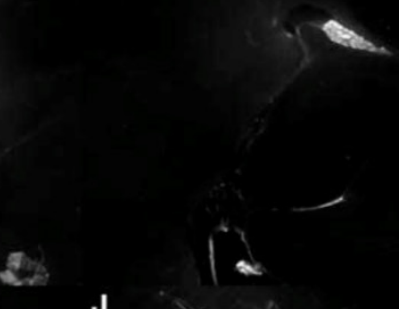

J
C

G
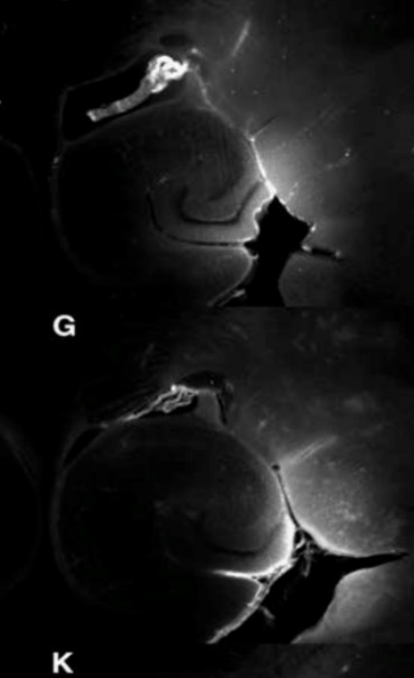

H

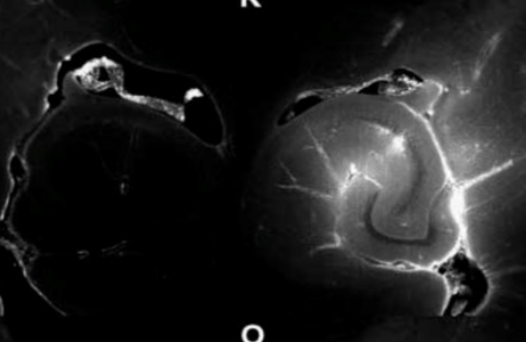

o

L

$\mathbf{P}$
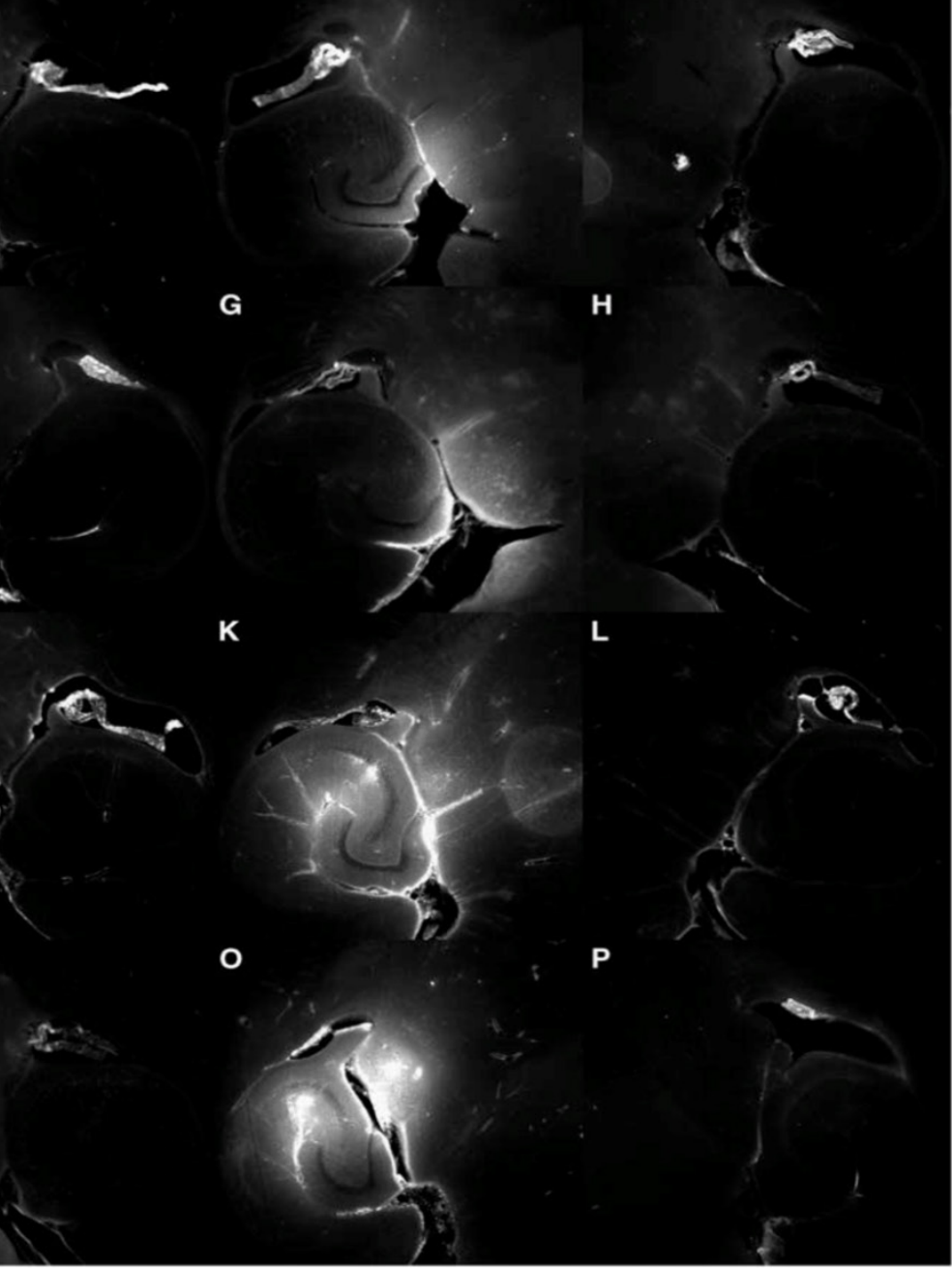

R
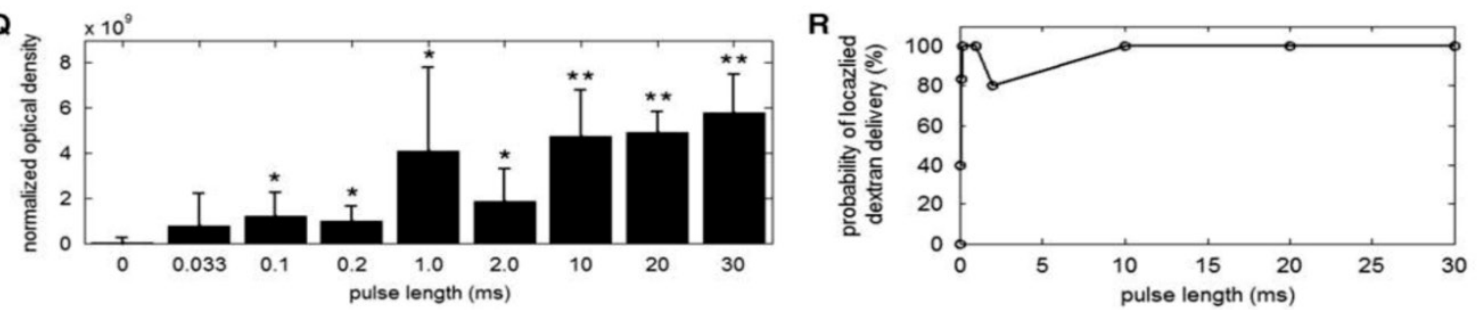

FIGURE 3. Qualitative fluorescence images of the (A, C, E, G, I, K, M, O) left and (B, D, F, H, J, L, N, P) right brain regions of interest (ROI) that have been exposed to pulse length (PL) of (A) 0.033, (C) 0.I, (E) 0.2, (G) I, (I) 2, (K) I0, (M) 20, and (O) 30 milliseconds. The white scale bar in (A) indicates I mm. Quantitative (Q) normalized optical density (NOD) of the left focused ultrasound (FUS)-targeted ROI and (R) probability of localized dextran delivery. The left ROI was sonicated at different PLs. The single asterisk $\left(^{*}\right)$ indicates an NOD increase from the sham, whereas the double asterisk $(* *)$ indicates a significant increase $(P<0.05)$ compared with the 0.033-, 0.1-, and 0.2-millisecond PLs [58].

\section{Molecular Delivery through the BBB open- ing}

A molecular delivery study [26, 52] indicated that the range of molecular size for trans-BBB delivery spreads to well beyond the 574 Da (Gadolinium; Fig. 2) to $67 \mathrm{KDa}$ (Albumin; Fig. 4) and $2000 \mathrm{kDa}$ (Dextrans; Fig. 4). As expected, at $2000 \mathrm{kDa}$ (or, $20 \mathrm{~nm}$ ), the fluorescent region is the smallest (since the mole- cule is the largest and thus diffusion the slowest) and mostly outside of the hippocampus. Therefore, FUS-induced BBB opening was shown feasible for noninvasive, local, and transient opening of the BBB for drug delivery of agents of several tens of $\mathrm{kDa}$; providing thus the opportunity of delivering available pharmacological agents to specific brain regions for treatment of neurological disease. 

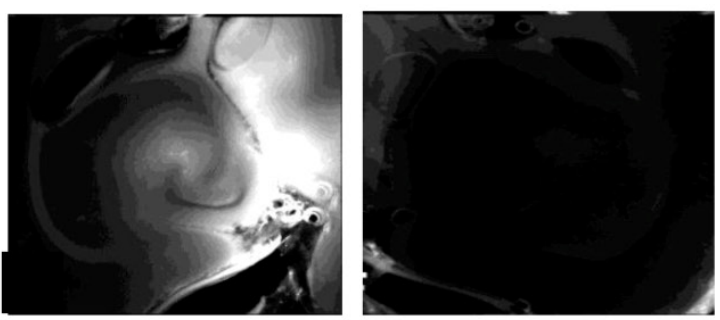

(i)
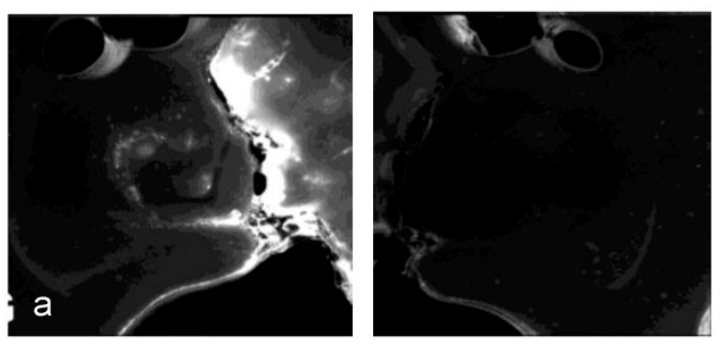

(ii)
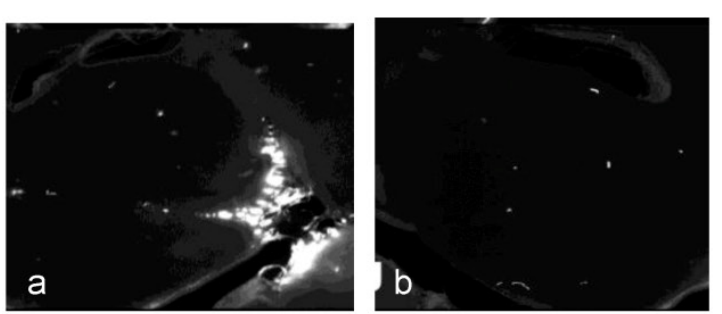

(iii)

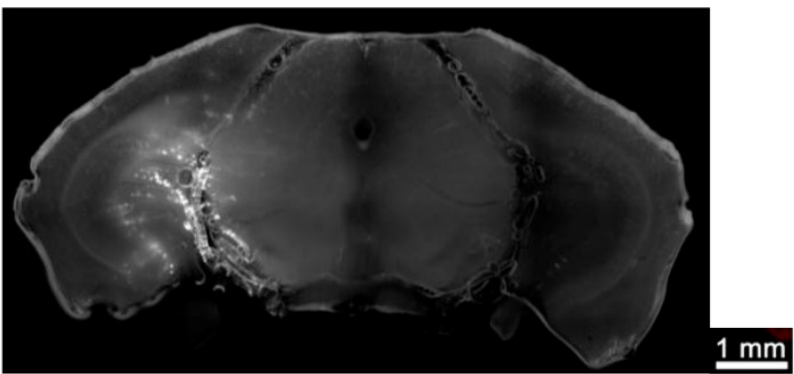

(iv)

FIGURE 4. Study of the molecular size through the BBB opening using Dextrans and fluorescence imaging: Horizontal slice of Dextran of molecular weight equal to i) 3 , ii) 70 and iii) $2000 \mathrm{kDa}$ on the a) left (targeted) and b) right (not targeted) hippocampus; iv) Coronal slice of the entire brain at 70 $\mathrm{kDa}$ Dextran showing the fluorescent left hippocampus (crescent-shaped); v) Fluorescent albumin $(67 \mathrm{kDa})$ permeated in the putamen through the opened BBB.

\section{Safety and reversibility of BBB opening}

In order to determine the safety window of the FUS technique, through histological and immunohistological techniques [27], in a preliminary study we have identified the safe operating parameters of ultrasound exposure for neurons, astrocytes, and endothelial cells (Fig. 5). Immunostaining studies to

confirm those results have also been reported in neurons [65] and are ongoing in the other cases. In summary, BBB opening starts occurring at $0.3 \mathrm{MPa}$ rarefactional pressure amplitude and beyond. At pressures under 0.6 MPa (Fig. 5(i)), no extravasation of red blood cells (RBC) or neuronal damage was observed in the regions of the hippocampus exhibiting the most pronounced BBB opening. Beyond 0.6 MPa (Fig. 5(ii)), RBC extravasation was detected and beyond $0.9 \mathrm{MPa}$ neuronal damage was observed. These preliminary findings suggest that there is overlap between the feasibility and safety windows within the pressure range of 0.3-0.6 $\mathrm{MPa}$, i.e., the $\mathrm{BBB}$ can be opened throughout the entire hippocampus without endothelial or neuronal damage at those pressures (Fig. 5; [25, 27]). FUS-induced BBB opening was reported to close within 24 hours in rabbits [10], mice [66] and monkeys [67]. Figure 6 shows that BBB closure had occurred within the first 24 hours after BBB opening.

Full assessment of the safety profile is more complex than the preliminary studies reported. Behavioral, cognitive, electrophysiological and additional cell histology are warranted for maximal safety profile delineation. Although the preliminary studies by our group and others have identified that stable cavitation of the bubbles is sufficient to open the blood-brain barrier (Fig. 7) and that histological sections indicate no damage (Fig. 3), both in vivo (fMRI, EEG and PET as well as cognitive studies) as well as ex vivo immunostaining of glia, astrocytes, pericytes and neurons still remain in order to fully describe the safety profile of the technique.
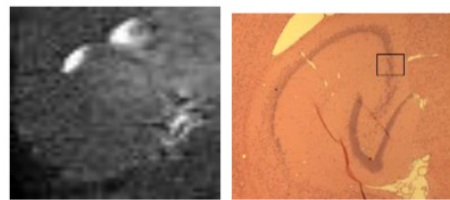

(i)
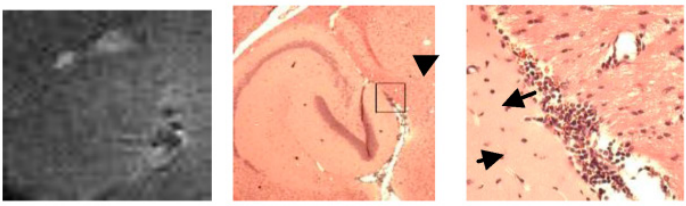

(ii)

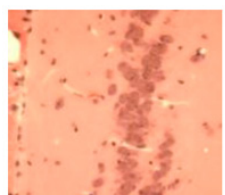

FIGURE 5. Comparison between MRI (left) and histology (center (IX) and right (200x near the region of most enhanced BBB opening according to the MRI) after FUS-induced BBB opening on the left hippocampus at i) 0.45 and ii) $0.75 \mathrm{MPa}$ peak rarefactional pressure. It shows that at lower pressures ((i)) the endothelial and neurons are intact (red) while at higher pressures ((ii)) there is extravasation of red blood cells (indicated by arrowhead) and neuronal death (indicated by arrow). This indicates the safety window of the FUS technique in BBB opening. 


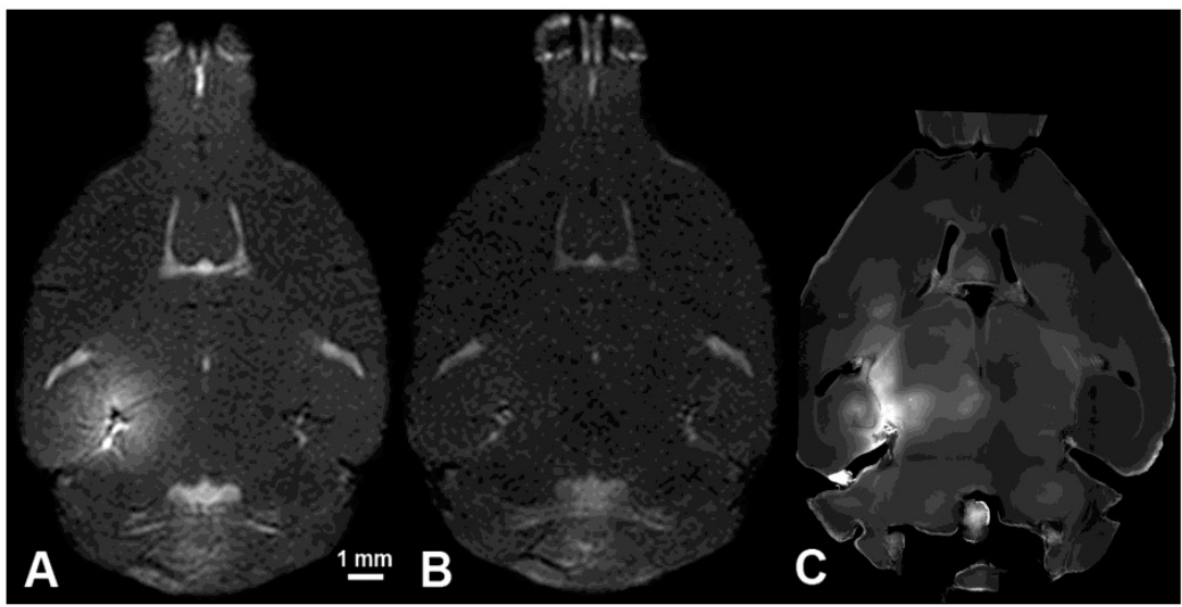

FIGURE 6. TI MRI images of A) BBB opening, B) BBB closing (24 hours); and C) fluorescence imaging with 3-kDa dextran of the left (sonicated hippocampus).

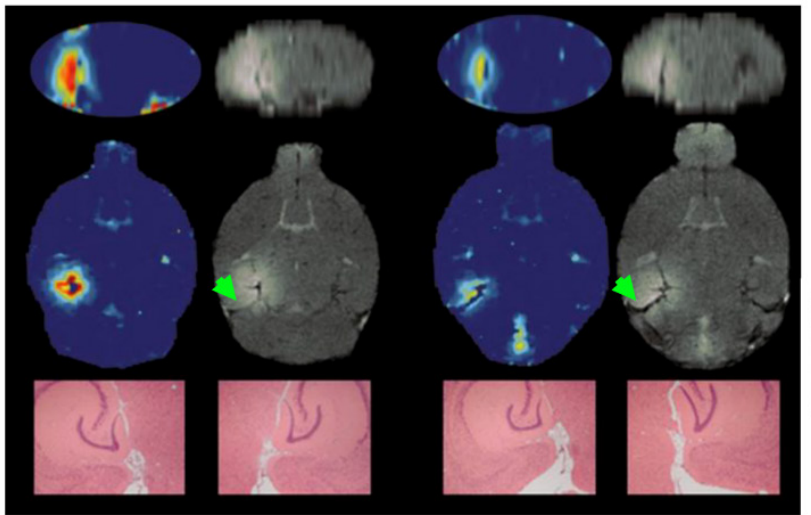

(i)

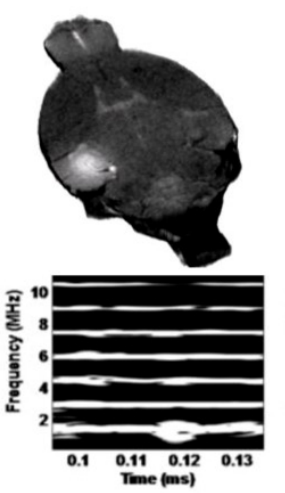

(ii)

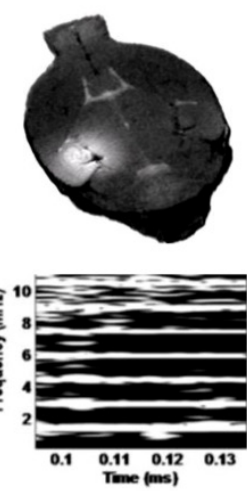

(iii)

FIGURE 7. MRI permeability image (first column in (i) and (ii)), TI-weighted image (first column in (i) and (ii)) of BBB opening in the left hippocampal formation (right one served as the control), H\&E histology of both the left and right hippocampi (40x) (last row in (i) and (ii)) and cavitation spectrograms (bottom row in (iii) with corresponding TI images on top) at $0.45 \mathrm{MPa}$ ((i) and right column in (iii) and $0.30 \mathrm{MPa}$ ((ii) and left column in (iii)). Note the harmonic peaks (parallel lines) in the spectrograms at I.5, 3, 4.5 MHz, etc at $0.3 \mathrm{MPa}$ and the inertial cavitation (harmonics and broadband noise) on the spectrogram at $0.45 \mathrm{MPa}$. No structural damage was noted at either pressure or cavitation phenomena in 48 mice studied [65]. The permeability maps show increase of a 100 fold in the area sonicated, i.e., the left hippocampal formation. The entorhinal cortex is designated using a green arrow. At higher pressures, damage was detected and therefore the highest pressure to be used in this study will be $0.45 \mathrm{MPa}$.

\section{Properties of BBB opening}

Dynamic contrast-enhanced (DCE)-MRI has been performed before and after the intraperitoneal injection of gadodiamide over $60 \mathrm{~min}$ [61]. The general kinetic model (GKM) is used to estimate the permeability in the entire brain [61]. At $0.3 \mathrm{MPa}$ and $4-5-\mu \mathrm{m}$ bubbles, the permeability is found to equal $0.02 \pm 0.0123 \mathrm{~min}-1$ and increase by at least 100 times in the region of $\mathrm{BBB}$ opening compared to the control side. Cavitation (Fig. 2) and permeability (Fig. 7) findings demonstrated that the inertial cavitation threshold is independent of the bubble size while both the ICD and MR amplitude increased at larger bubble sizes, also indicating a correlation between the cavitation and permeability increase [61]. The fact that the permeability increased with the pressure and microbubble size indicated that the BBB opening occurs at multiple sites within the capillary tree and that the BBB opening is larger with larger microbubbles, most likely due to the larger area of contact between the bubble and the capillary wall.

\section{BBB opening in large animals}

A 3D finite-difference, time-difference simulation platform (Wave 3000, CyberLogic, New York, USA) simulation model, validated in experiments (Fig. 8(c); [62]) was used to identify the optimal frequency for successful trans-skull propagation using CT scans (GE LightSpeed Ultrafast CT scanner; available in the department of radiology at) of ex vivo non-human primate and human skulls as inputs to 
model absorption and speed of sound maps. The targeted brain structures were extracted from publicly available 3D brain atlases registered with the skulls (Fig. 8(a-b)). The frequency of $500 \mathrm{kHz}$ provided the best tradeoff between phase aberrations and standing wave effects in the human case while the frequency of $800 \mathrm{kHz}$ was most suitable in the case of the primate skull. A fast periodic linear chirp method was devel-

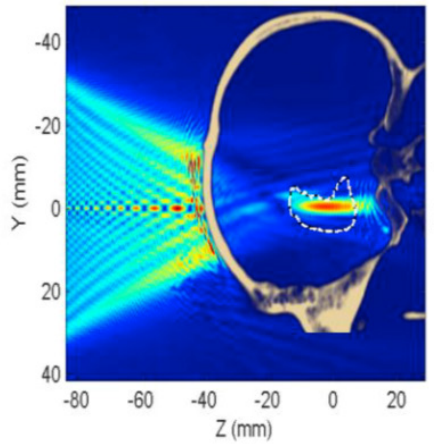

(a)

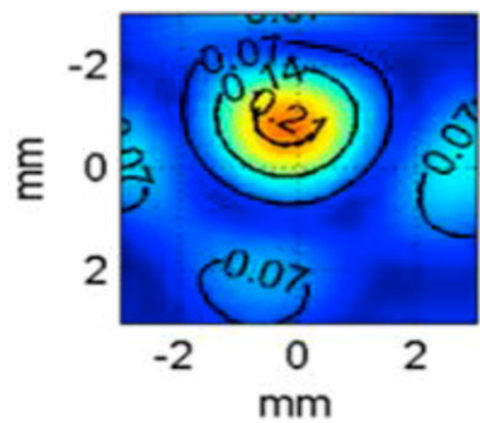

(c) oped and found capable of reducing the standing wave effects. The simple, single-element system that we have been using in mice was concluded to be feasible for BBB opening in primates and humans and the size of the focal spot dimensions fit the hippocampal sizes when targeting through the dorsal part of the skull (Fig. 9).

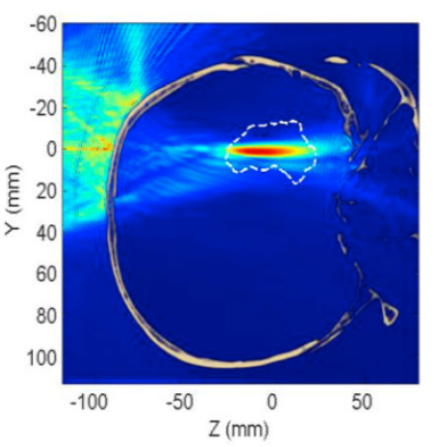

(b)

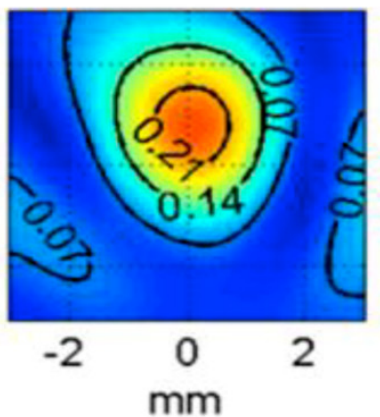

(d)

FIGURE 8, Theoretical simulations with experimental validation for predicting the area of BBB opening (in red) relative to the hippocampus (white dashed contour through the skull) of a) non-human primates at $800 \mathrm{kHz}$ and b) human at $500 \mathrm{kHz}$. In both cases, there is formation of a uniform focal spot with the largest dimension along the longest dimension of the hippocampus in both cases. c) Experimental validation of a uniform focal spot (transverse view) through the ex vivo primate skull of the d) simulated focal spot at $800 \mathrm{kHz}$ [62].
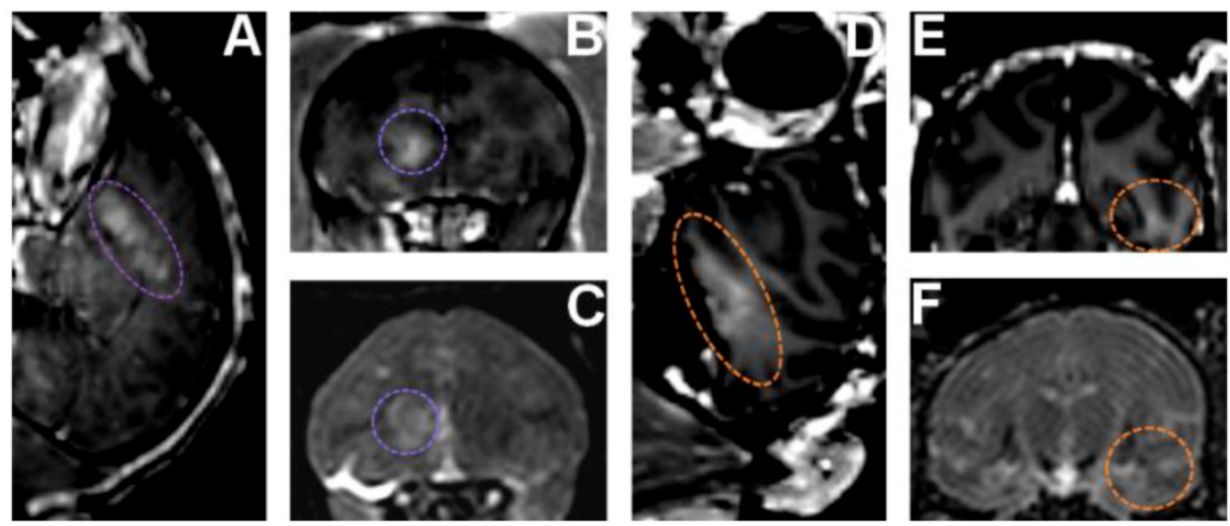

FIGURE 9. In vivo BBB opening in monkeys: (A,B,C) BBB opening experiment targeting the caudate using custom made microbubbles and applying $0.6 \mathrm{MPa}$ (purple dashed line shows region of interest). (D,E,F) BBB opening experiment targeting hippocampus using Definity ${ }^{\circledR}$ microbubbles and applying $0.6 \mathrm{MPa}$ (orange dashed line shows region of interest). (A,B,D,E) 3D Spoiled Gradient-Echo (SPGR) TI-weighted sequence was applied after intravenous (IV) injection of gadodiamide I h after sonication. (A,D) Sagittal slices at the region of interest. (B,E) Corresponding coronal slices. (C,F) 3D T2-weighted sequence, an edema was visible using custom made microbubbles while no damage was detected using Definity® microbubbles. 


\section{Therapeutic delivery through the blood-brain barrier opening}

Neurotrophic delivery to the brain has been proven essential in reversing the neuronal degeneration process but so far has been hindered by the blood-brain barrier. In a recent study by our group, not only was it shown that the brain-derived neurotrophic factor (BDNF) can cross the ultrasound-induced blood-brain barrier opening but also that it can trigger signaling pathways in the pyramidal neurons of mice in vivo from the membrane to the nucleus (Fig. 10) [65]. This opens entirely new avenues in the brain drug delivery where focused ultrasound in conjunction with microbubbles can generate downstream effects at the cellular and molecular level and thus increase the drug's efficacy and potency in controlling or reversing the disease.

FIGURE 10. (a) Fluorescent image of a 100-micron frozen brain section from a mouse that was sacrificed $20 \mathrm{~min}$ after sonication. The sonicated hippocampus (left) shows much higher fluorescent intensity than the un-sonicated hippocampus (right), depicting blood-brain barrier opening and the extravasation of fluorescent-tagged (Alexa Fluor 594) BDNF in the sonicated region; (b) a 5-micron frozen section from the same mouse was immunohistochemically stained using a primary antibody against phosphorylated MAPK (PMAPK). Consistent with the fluorescent image in (a), the intensity of DAB staining is much greater in the left sonicated hippocampus compared to the right control; the black box shows the enlarged area in (c), where immunoreactivity to PMAPK is shown in mossy fiber terminals (arrowhead), suprapyramidal CA3 dendrites (black star), and the axons of the Schaffer collateral system (hollow star); (d) immunohistochemical staining of a 5-micron frozen section from a mouse that was sacrificed $3 \mathrm{~min}$ after sonication; the same primary antibody against PMAPK was used. No difference in DAB intensity is observed between the sonicated and the control hippocampus; (e) Negative control for the same mouse in (a); no primary antibody (against pMAPK) was added to this 5 -micron frozen section during the staining procedure. All magnifications are $40 x$ and scale bars are $500 \mu \mathrm{m}$ except for (c), which is $100 x$ and $200 \mu \mathrm{m}$, respectively. In ( $f$ ), immunohistology stain intensity analysis shows percentage change between the left (FUS) and the right (no FUS) sides of the mice brains. A significant difference ( $<<0.05, N=3$; depicted by asterisks) was found between the BDNF administered animal group and the control (no BDNF) animal group for the TrkB, MAPK, and CREB antibodies. Bars represent mean \pm standard deviation.

\section{DISCUSSION}

Despite the fact that FUS is currently the only technique that can open the BBB locally and noninvasively (Table 1), several key aspects such as those proposed to be fully investigated in this study had remained incomplete. Although the presence of microbubbles allows for a reduction in the necessary acoustic pressure for BBB opening, it also increases the probability of disrupting the microbubble through inertial cavitation. Not only can the resulting effects open the tight junctions, but also induce irreversible
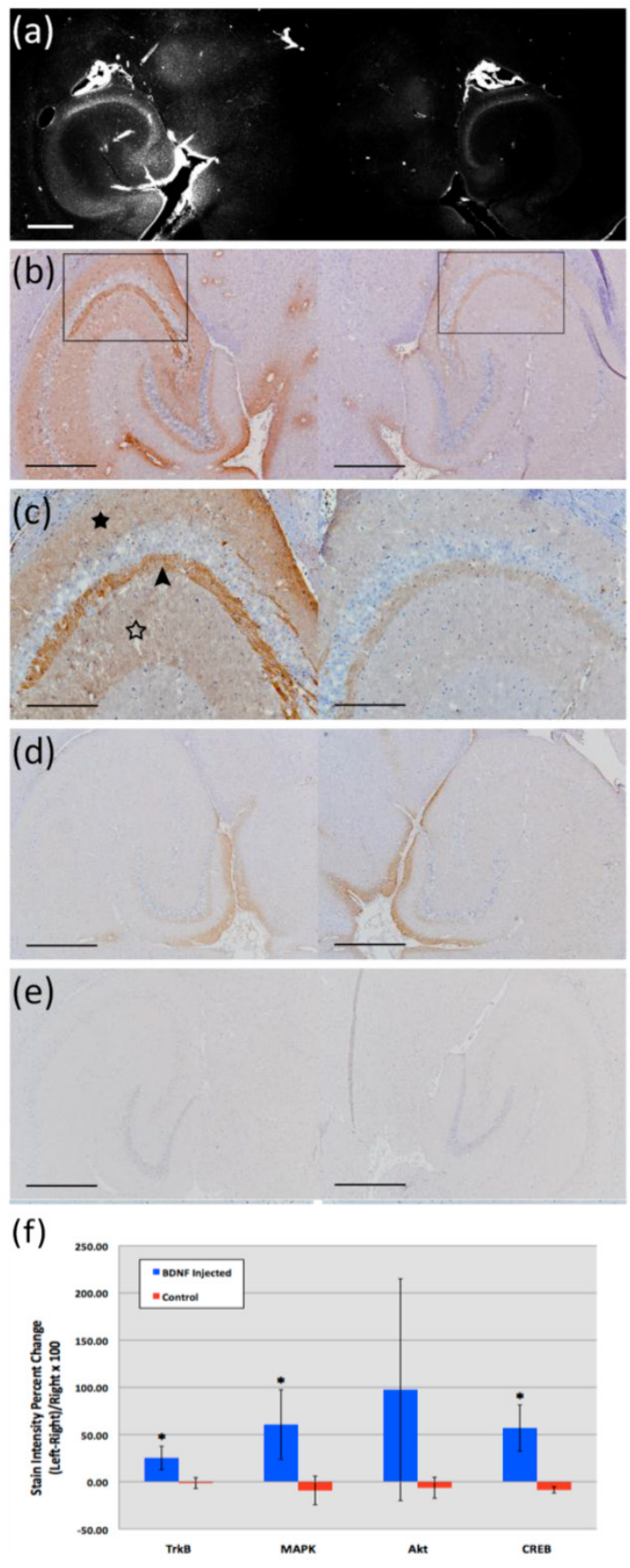

damage to the blood vessels and its surrounding cells. Previous studies by other groups explore the brain as a whole and attempt to induce BBB opening in arbitrary, multiple locations without targeting a specific brain region such as the hippocampus and evaluating its properties locally. Furthermore, multi-element phased arrays (with up to 1024 elements) that permit phase aberration correction have been proposed in order to increase flexibility of the location targeted, mainly used for tumor ablation with minimal aberra- 
tion. However, these arrays are highly complex and difficult to manufacture and cumbersome in handling and positioning around a subject due to their typically bulky size and weight. Finally, the delivery of many large agents using focused ultrasound (FUS) and microbubbles had been demonstrated in previous studies by our group and others. However, despite the promise shown by the delivery of such a variety of compounds, several questions on the effectiveness of the delivery remain. More specifically, it is still not known whether therapeutic molecules can cross through the BBB opening into the intracellular neuronal space so that they can trigger the required downstream effects for neuronal regeneration.

Given the established feasibility of FUS in BBB opening and its urgent need in brain drug delivery, the main findings summarized in this paper are as follows:

1) The BBB can be reproducibly opened in a specific subcortical region associated with neurodegenerative disease, i.e., the hippocampus.

2) BBB opening can be induced without requiring craniotomy or MRI for targeting in mice and monkeys.

3) A single-element transducer is sufficient to induce trans-skull wave propagation through both mouse and human skulls ex vivo and can lead to a well-formed focal spot for BBB opening in both cases but at distinct ultrasound frequencies. More importantly, unlike tumor ablation using multi-element arrays, lower pressures and anatomy-specific (not tumor-specific) targeting are required, potentially rendering sonication with a single-element transducer sufficient.

4) An optimization study has been performed that has identified the pulse length and peak-rarefactional pressure range, within which $\mathrm{H} \& \mathrm{E}$ studies have shown that there is no structural damage associated with BBB opening has been identified. This is very similar to ultrasound imaging, which has been proven safe according to a specific range of pulse lengths and pressures used.

5) Preliminary delivery of molecules on the order of 0.5-70 kDa, including Dextran and BDNF molecules, has been shown to be successfully delivered and localized within the intracellular space of neurons triggering a specific signaling pathway.

6) Intraperitoneal (IP)-administered gadolinium allowed for spatio-temporal analysis of the $\mathrm{BBB}$ opening with MRI. The BBB permeability could thus be assessed in vivo and shown to increase by at least a 100 -fold in the targeted hippocampus.

\section{CONCLUSION}

The FUS in conjunction with microbubbles was hereby shown to effectively and reproducibly open the blood-brain barrier transcranially in vivo with its recovery occurring within the first 24 hours. The permeability of the FUS-opened BBB was shown to increase by at least two orders of magnitude indicating facilitation of drug delivery through FUS. Molecules of a wide range in sizes were capable of traversing the opened BBB without any associated structural damage. A dependence of the BBB permeability on the pressure and the microbubble size indicated that multiple sites of BBB opening within the ultrasound beam occur simultaneously while each BBB opening site increases with the microbubble size. Finally, a new pulse sequence was designed that showed feasibility at very short pulse lengths and transcranial BBB opening in larger animals, such as non-human primates and humans, was shown feasible in simulations and ex vivo experiments as well as in vivo primate monkeys (Fig. 9) [63].

\section{ACKNOWLEDGEMENTS}

Work shown here was performed by Yao-Sheng Tung, James Choi, Thomas Deffieux, Babak Baseri, Fabrice Marquet and Fotios Vlachos in the Ultrasound and Elasticity Imaging Laboratory at Columbia. Collaborators on the microbubble studies were Jameel Feshitan and Mark Borden (currently at the University of Colorado-Boulder) and on the primate studies Tobias Techert and Vincent Ferrera (Columbia University). The research was supported by NIH R01 EB009041, NIH R21 EY018505, NSF CAREER 064471, the Kinetics Foundation and the Kavli Institute.

\section{Competing Interests}

The authors have declared that no competing interest exists.

\section{References}

[1] Pardridge WM. The blood-brain barrier: bottleneck in brain drug development. NeuroRx 2005; 2: 3-14.

[2] Abbott NJ, Ronnback L, and Hansson E. Astrocyte-endothelial interactions at the blood-brain barrier. Nat Rev Neurosci 2006; 7: 41-53.

[3] Stewart P Aand Tuor UI. Blood-eye barriers in the rat: correlation of ultrastructure with function. J Comp Neurol 1994; 340: 566-76.

[4] Pardridge WM. Molecular trojan horses for blood-brain barrier drug delivery. Curr Opin Pharmacol 2006; 6(5):494-500.

[5] Bakay L, Ballantine HT Jr, Hueter TF, et al. Ultrasonically produced changes in the blood-brain barrier. AMA Arch Neurol Psychiatry 1956; 76:457-67.

[6] Ballantine HT Jr, Bell E, and Manlapaz J. Progress and problems in the neurological applications of focused ultrasound. J Neurosurg 1960;17:858-76.

[7] Patrick JT, Nolting MN, Goss SA, et al. Ultrasound and the blood-brain barrier. Adv Exp Med Biol 1990; 267: 369-81.

[8] Vykhodtseva NI, Hynynen K, and Damianou C. Histologic effects of high intensity pulsed ultrasound exposure with subharmonic emission in rabbit brain in vivo. Ultrasound Med Biol 1995; 21: 969-79. 
[9] Mesiwala AH, Farrell L, Wenzel HJ S, et al. High-intensity focused ultrasound selectively disrupts the blood-brain barrier in vivo. Ultrasound Med Biol 2002; 28: 389-400.

[10] Hynynen K, McDannold N, Vykhodtseva N, et al. Noninvasive MR imaging-guided focal opening of the blood-brain barrier in rabbits. Radiology 2001; 220: 640-6.

[11] Hynynen K, McDannold N, Vykhodtseva N, et al. Non-invasive opening of BBB by focused ultrasound. Acta Neurochir Suppl 2003; 86: 555-8.

[12] McDannold N, King RL, and Hynynen K. MRI monitoring of heating produced by ultrasound absorption in the skull: in vivo study in pigs. Magn Reson Med 2004; 51: 1061-5.

[13] Sheikov N, McDannold N, Vykhodtseva N, et al. Cellular mechanisms of the blood-brain barrier opening induced by ultrasound in presence of microbubbles. Ultrasound Med Biol 2004; 30: 979-89.

[14] Hynynen K, McDannold N, Sheikov NA, et al. Local and reversible blood-brain barrier disruption by noninvasive focused ultrasound at frequencies suitable for trans-skull sonications. Neuroimage 2005; 24: 12-20.

[15] McDannold N, Vykhodtseva N, Raymond S, et al. MRI-guided targeted blood-brain barrier disruption with focused ultrasound: histological findings in rabbits. Ultrasound Med Biol 2005; 31: 1527-37.

[16] Hynynen K, McDannold N, Vykhodtseva N, et al. Focal disruption of the blood-brain barrier due to $260-\mathrm{kHz}$ ultrasound bursts: a method for molecular imaging and targeted drug delivery. J Neurosurg 2006; 105 : 445-54.

[17] McDannold N, Vykhodtseva N, and Hynynen K. Targeted disruption of the blood-brain barrier with focused ultrasound: association with cavitation activity. Phys Med Biol 2006; 51: 793-807.

[18] Sheikov N, McDannold N, Jolesz F, et al. Brain arterioles show more active vesicular transport of blood-borne tracer molecules than capillaries and venules after focused ultrasound-evoked opening of the blood-brain barrier. Ultrasound Med Biol 2006; 32: 1399-409.

[19] Choi JJ, Pernot M, Small SA, et al. Feasibility of transcranial, localized drug-delivery in the brain of Alzheimer's-model mice using focused ultrasound. IEEE Proc Inter Ultrasonics Symposium 2005;:988-991.

[20] Choi JJ, Pernot M, Small S, et al. Noninvasive Blood-Brain Barrier Opening in Live Mice. AIP Conference Proceedings 2006; 829:271-275.

[21] Choi JJ, Pernot M, Brown T R, et al. Spatio-temporal analysis of molecular delivery through the blood-brain barrier using focused ultrasound. Phys Med Biol 2007; 52:5509-30.

[22] Choi JJ, Pernot M, Small SA, et al. Noninvasive, transcranial and localized opening of the blood-brain barrier using focused ultrasound in mice. Ultrasound Med Biol 2007; 33: 95-104.

[23] Konofagou EE, Choi J, Baseri B, et al. Characterization and Optimization of Trans-Blood-Brain Barrier Diffusion In Vivo. Minneapolis, MN, USA: 8the International Symposium on Therapeutic Ultrasound. $2009: 418-422$.

[24] Choi JJ, Wang S, Brown TR, et al. Noninvasive and transient blood-brain barrier opening in the hippocampus of Alzheimer's double transgenic mice using focused ultrasound. Ultrason Imaging 2008; 30: 189-200.

[25] Konofagou E and Choi JJ. Ultrasound-induced treatment of neurodegenerative diseases across the blood-brain barrier. Biomedical Applications of vibration and Acoustics in therapy, Bioeffects and modelling, A A-J a M Fatemi, Ed New York: ASME Press 2008: 63-80.

[26] Choi JJ, Wang S, Tung YS, et al. Molecules of various pharmacologically-relevant sizes can cross the ultrasound-induced blood-brain barrier opening in vivo. Ultrasound Med Biol 2010; 36 (1): 58-67.

[27] Baseri B, Choi JJ, Tung YS, et al. Safety assessment of blood-brain barrier opening using focused ultrasound and definity microbubbles: a short-term study. Ultras Med Biol 2010; 36(9): 1445-1459.

[28] Kaufmann BA, Wei K, and Lindner JR. Contrast Echocardiography. Curr Probl Cardiol 2007; 32: 51-96.

[29] Christensen DA. Ultrasonic Bioinstrumentation. New York: John Wiley \& Sons. 1988

[30] Miller DL. Overview of experimental studies of biological effects of medical ultrasound caused by gas body activation and inertial cavitation. Progress in Biophysics \& Molecular Biology 2007; 93: 314-30.

[31] Chen S, Kroll MH, Shohet RV, et al. Bioeffects of myocardial contrast microbubble destruction by echocardiography. Echocardiagraphy 2002; 19: 495-500.

[32] Li P, Armstrong WF, Miller DL. Impact of myocardial contrast echocardiography on vascular permeability: Comparison of three different contrast agents. Ultrasound Med Biol 2004; 30: 83-91.

[33] Li P, Cao LQ, Dou CY, et al. Impact of myocardial contrast echocardiography on vascular permeability: An in vivo dose response study of delivery mode, pressure amplitude and contrast dose. Ultrasound Med Biol 2003; 29: 1341-9.
[34] Kaps M, Seidel G, Algermissen C, et al. Pharmacokinetics of echocontrast agent infusion in a dog model. Journal of Neuroimaging 2001; 11: 298-302.

[35] Liu GY, Tan K, et al. Impact of microbubble enhanced ultrasound on blood-brain barrier permeability: An in vivo dose response study of ultrasound intensity and contrast dose. Circulation 2005; 112: U602-U605.

[36] Yang FY, Fu WM, Yang RS, et al. Quantitative evaluation of focused ultrasound with a contrast agent on blood-brain barrier disruption. Ultrasound Med Biol 2007; 33: 1421-7

[37] Miller D, Li P, Dou C, et al. Influence of contrast agent dose and ultrasound exposure on cardiomyocyte injury induced by myocardial contrast echocardiography in rats. Radiology 2005; 237: 137-143

[38] Christiansen J, French BA, Klibanov AL, et al. Targeted tissue transfection with ultrasound destruction of plasmid-bearing cationic microbubbles. Ultrasound Med Biol 2003; 29:1759-67.

[39] Chomas JE, Dayton P, May D, et al. Threshold of fragmentation for ultrasonic contrast agents. J Biomed Opt 2001; 6: 141-50.

[40] Borden M, Kruse D, Caskey C, et al. Influence of lipid shell physicochemical properties on ultrasound-induced microbubble destruction. IEEE Trans Ultrason Ferroelect Freq Contr 2005; 52: 1992-2002.

[41] Iadecola C. Neurovascular regulation in the normal brain and in Alzheimer's disease. Nat Rev Neurosci 2004; 5: 347-360.

[42] Ghose A, Viswanadhan VN, Wendoloski JJ. A knowledge-based approach in designing combinatorial or medicinal chemistry libraries for drug discovery 1 A qualitative and quantitative characterization of known drug databases. J Comb Chem 1999; 1: 55-68.

[43] Lipinski C. Drug-like properties and the causes of poor solubility and poor permeability. J Pharmacol Toxicol Methods 2000; 44: 235-249.

[44] Fischer H, Gottschlich R, Seelig A. Blood-brain barrier permeation: molecular parameters governing passive diffusion. J Membr Biol 1998; 165: 201-11.

[45] Blasberg R, Patlak C, Fenstermacher JD. Intrathecal chemotherapy brain tissue profiles after ventriculocisternal perfusion. J Pharmacol Exp Ther 1975; 195: 73-83.

[46] Fung L, Shin M, Tyler B, et al. Chemotherapeutic drugs released from polymers distribution of 1,3-bis(2-chloroethyl)-1-nitrosourea in the rat brain. Pharm Res 1996; 13: 671-682.

[47] Pardridge WM. Drug targeting to the brain. Pharm Res 2007; 24: 1733-44.

[48] Neppiras EA. Acoustic cavitation. Physics Reports 1980, 61: 159-251.

[49] Leighton T. The Acoustic Bubble. First ed London: Academic, 1994.

[50] Choi JJ, Feshitan JA, Wang S, et al. The Dependence of the Ultrasound-Induced Blood-Brain Barrier Opening Characteristics on Microbubble Size In Vivo. Minneapolis, MN, USA: 8the International Symposium on Therapeutic Ultrasound., 2009:58-62.

[51] Choi JJ, Small SA, Konofagou EE. Optimization of Blood-Brain Barrier Opening in Mice using Focused Ultrasound. IEEE Proc InternUltrasonics Symposium 2006; : 540-543.

[52] Choi JJ, Wang S, Morrison IIIB, et al. Focused Ultrasound-Induced Molecular Delivery through the Blood-Brain Barrier. EEE Proc Intern Ultrasonics Symposium 2007; :1192-1195.

[53] Kinoshita M, McDannold NF, Jolesz A, et al. Noninvasive localized delivery of Herceptin to the mouse brain by MRI-guided focused ultrasound-induced blood-brain barrier disruption. Proc Natl Acad Sci U S A 2006; 103: 11719-23.

[54] Raymond SB, Treat LH, Dewey JD, et al. Ultrasound enhanced delivery of molecular imaging and therapeutic agents in Alzheimer's disease mouse models. PLoS ONE 2008; 3: e2175.

[55] Sheikov N, McDannold N, Sharma S, et al. Effect of Focused Ultrasound Applied With an Ultrasound Contrast Agent on the Tight Junctional Integrity of the Brain Microvascular Endothelium. Ultrasound Med Biol 2008; 34(7):1093-104.

[56] Treat LH, McDannold N, Vykhodtseva N, et al. Targeted delivery of doxorubicin to the rat brain at therapeutic levels using MRI-guided focused ultrasound. Int I Cancer 2007; 121: 901-7.

[57] Feshitan JA, Chen CC, Kwan JJ, et al. Microbubble size isolation by differential centrifugation. J Colloid Interface Sci 2009; 329: 316-24.

[58] Choi J, Selert K, Vlachos F, et al. Noninvasive and localized neuronal delivery using short ultrasonic pulses and microbubbles. Proc Natl Acad Sci U S A 2011;108(40):16539-44.

[59] Farny CH, Holt RG, and Roy RA. Temporal and spatial detection of HIFU-induced inertial and hot-vapor cavitation with a diagnostic ultrasound system. Ultrasound Med Biol 2009; 35: 603-15.

[60] Tung Y, Vlachos F, Selert K, et al. In vivo Transcranial Cavitation Threshold Detection during Ultrasound-Induced Blood-Brain Barrier Opening. Phys Med Biol 2010; 55(20): 6141-6155. 
[61] Vlachos F, Tung YS and Konofagou EE. Permeability assessment of the focused ultrasound-induced blood-brain barrier opening using dynamic contrast-enhanced MRI. Phys Med Biol 2010; 55: 5451-5466.

[62] Deffieux TA, Konofagou EE. Numerical study and experimental validation of a simple transcranial focused ultrasound system applied to Blood-Brain Barrier Opening. IEEE-UFFC Trans 2010; 57(12): 2637-53

[63] Marquet F, Tung YS, Teichert T, et al. Noninvasive, transient and selective blood-brain barrier opening in non-human primates in vivo. Plos One 2011; 6(7):e22598.

[64] Tung YS, Vlachos F, Feshitan J, et al. The Mechanism of the Interaction between Focused Ultrasound and Microbubbles in Blood-Brain Barrier Opening in Mice. J Acous Soc Amer 2011; 108(40): 3059-3067.

[65] Baseri B, Choi JJ, Deffieux T, et al. Activation of signaling pathways following localized delivery of systemically administered neurotrophic factors across the blood-brain barrier using focused ultrasound and $\mathrm{mi}-$ crobubbles. Phys Med Biol 2012; 57: N65-81.

[66] Howles GP, Bing KF, Qi Y, et al. Contrast-enhanced in vivo magnetic resonance microscopy of the mouse brain enabled by noninvasive opening of the blood-brain barrier with ultrasound. Magn Reson Med 2010; 64(4):995-1004.

[67] McDannold N, Arvanitis CD, Vykhodtseva N, et al. Temporary disruption of the blood-brain barrier by use of ultrasound and microbubbles: safety and efficacy evaluation. rhesus macaques Cancer Res 2012; 72(14):3652-632012.

[68] Wang PH, Liu HL, Hsu PH, et al. Gold-nanorod contrast-enhanced photoacoustic micro-imaging of focused-ultrasound induced blood-brain-barrier opening in a rat model. J Biomed Opt 2012; 17(6):061222.

[69] Arvanitis CD, Livingstone MS, Vykhodtseva N, et al. Controlled Ultrasound-Induced Blood-Brain Barrier Disruption Using Passive Acoustic Emissions Monitoring. PloS One 2012; 7 (9): e45783.

[70] Burgess A, Ayala-Grosso CA, Ganguly M, et al. Targeted delivery of neural stem cells to the brain using MRI-guided focused ultrasound to disrupt the blood-brain barrier. PLoS One 2011; 6(11):e27877. 